\title{
The Influence of Parameters on the Generatrix of the Helicoid Form Guide of Geokhod Bar Working Body
}

\author{
Vladimir Aksenov ${ }^{1,3^{*}}$ Vladimir Sadovets ${ }^{2,3}$, and Dmitriy Pashkov ${ }^{1,3}$ \\ ${ }^{1}$ Institute of Coal of the Federal Research Center Coal and Coal Chemistry Siberian Branch of the RAS, \\ 650610, Leningradsky av, Kemerovo, Russia \\ ${ }^{2}$ T.F. Gorbachev Kuzbass State Technical University, 650000, 28 Vesennyaya St., Kemerovo, Russia \\ ${ }^{3}$ Yurga Technological Institute branch of Tomsk Polytechnic University, 652052 Leningradskay Str. \\ 26, Yurga, Russia.
}

\begin{abstract}
Influence of geometrical parameters of generatrix of helicoid on a guide of geokhod bar working body is proved in article. The relevance of the conducted research is considered and proved. General characteristics of the geokhod are presented. Features of geokhod working body, in particular formation of irregular shape of a surface of a face and working body are formulated and also it is told that at screw movement of geokhod working body of a face, points of working body will be formed a helicoid (screw) surface of a face. For establishing of the goals and objectives of research general geometrical parameters of generatrix is marked and justified which treat length of generatrix, width of generatrix of helicoid, pitch of helicoid and it's form. Forms of guides of geokhod bar working body based on basis parameters of geokhod and accepted general geometrical parameters of geokhod working body are received and presented. In virtue of the conducted research the dependence of a form of a guide of bar on general geometrical parameters of helicoid is defined and also basis parameters of helicoid in influencing a form of guide of working body.
\end{abstract}

\section{Introduction}

Because of increase of the number of vehicles and intensive growth of the population in the cities the critical shortage of territories is observed. In this regard in many large settlements of the world experts actively master underground space. However formation of a cavity in underground space with use of the tunnelling equipment causes need of the solution of questions of safety of carrying out mining operations, increases speed of a driving and labor productivity, decreases in prime cost $[1,2,3,4]$.

The perspective direction which is solved this question is using of geokhod technology for formation of a cavity in underground space where basic element is the geokhod $[5,6,7]$.

The geokhods belong to the new class of the driving equipment. Distinctive feature of the geokhods is screwing of container to the rock mass. So power crowding are created as a

* Corresponding author:55vva42@,mail.ru 
result of introduction of elements of the propulsor to the peripheric massif and uses of the normal reaction arising at interaction of elements of the propulsor of the geokhod with the massif of rocks. At present, a prototype of geokhod is manufactured with the cutting body for destruction of rocks of medium hardness (Figure 1) [8,9,10,11].

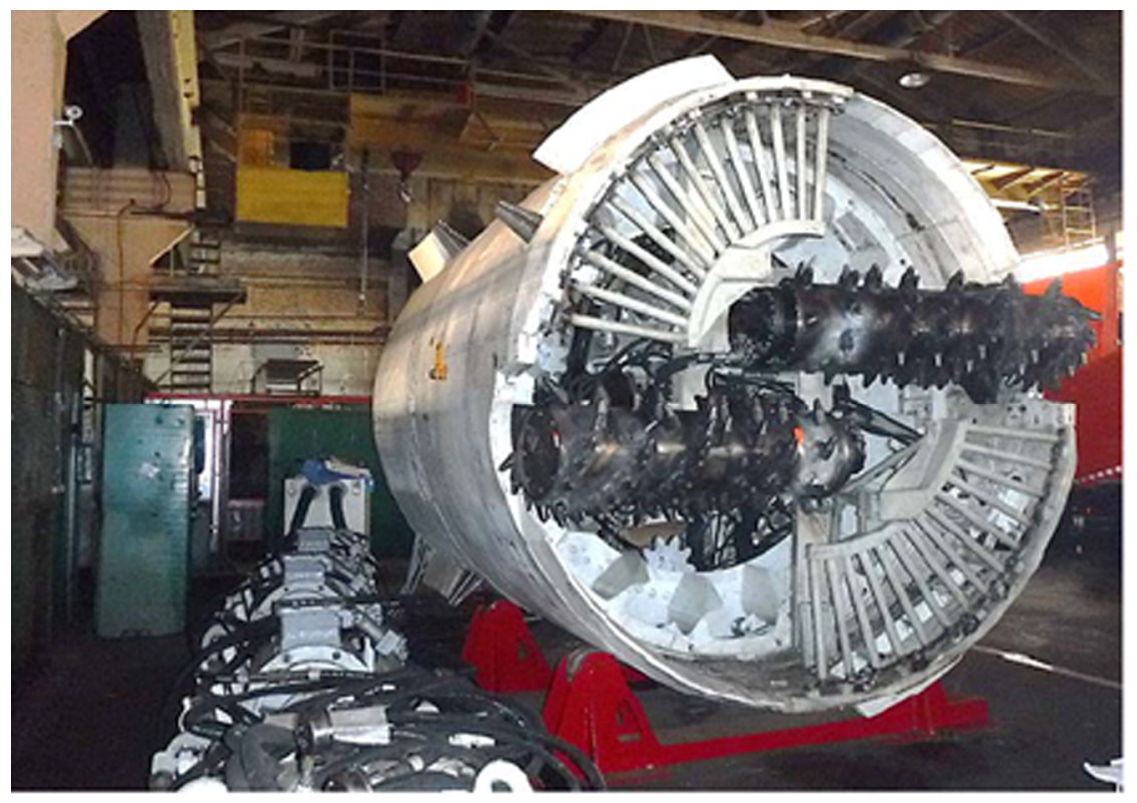

Fig. 1. Geokhod prototype with drum cutting body (CB).

At the existing development stage of elements of geokhod technology is particularly acute need in development of constructive and technical solutions of working body which is capable to carry out formation of a cavity in underground space on grounds with a hardness up to 1 on M.M. Protodyakonov's scale [12].

\section{Materials and methods}

Features of geokhod working body. A peculiar features of movement of the geokhod of a face causes formation of irregular shape of a surface not only the face, but also the working body (WB). A face surface, at destruction of his WB of the geokhod, has an appearance of several helicoid surfaces with ledges.

Any point of WB located at distance $\mathrm{x}$ (figure 2) from pivot of the geokhod moves to a face at an angle[7]:

$$
\beta_{x}=\operatorname{arctg} \frac{h_{B}}{2 \pi x} .
$$

Points of WB which are closer to pivot of the geokhod move to a face at an bigger angle, than the points which are on the periphery. Thus, at screw movement of WB of the geokhod of a face of a point of WB forms a helicoid (screw) surface. Therefore, the site of a surface of a face in the sector between adjacent beams after their pass takes a form of a screw surface. 


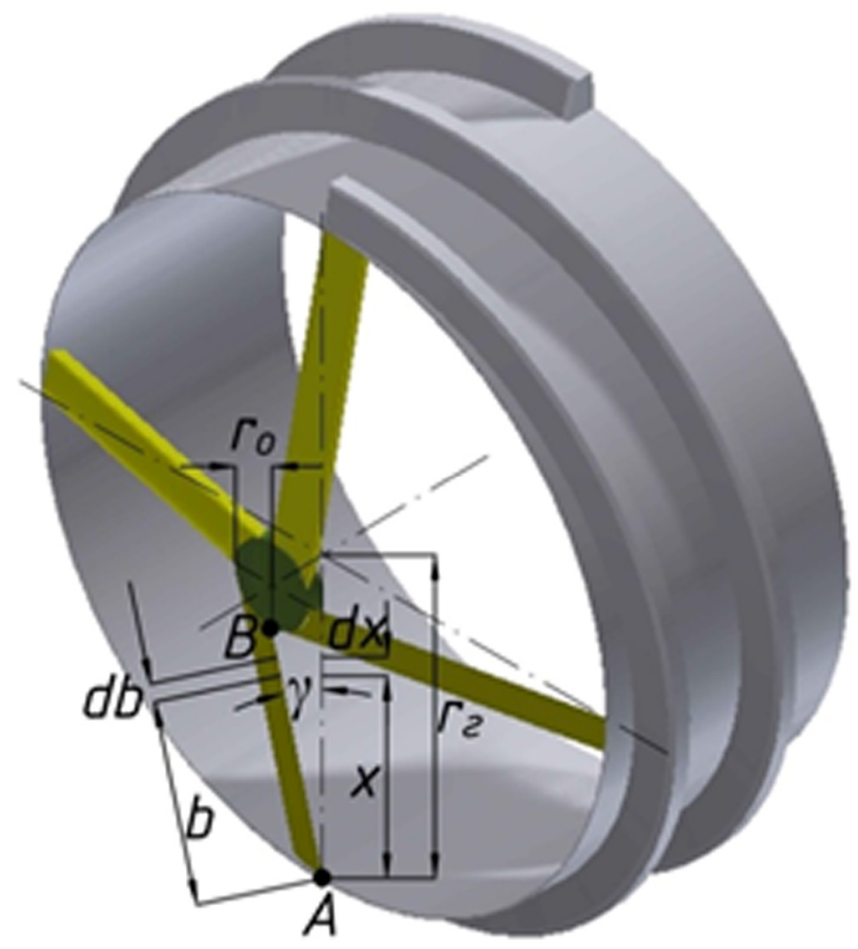

Fig. 2. Scheme of geokhod working body.

It agrees developing technical solutions, on the basis of a structural portrait of the geokhod, beams of WB can be at an some angle to pivot of the geokhod. The corner y (figure 1) can be: equal 90 angle degree, less than 90 angle degree - WB takes a form of a right cone, more than 90 angle degree - WB will be in the form of the back taper [7].

On the basis above stated the problem of the presented research is formulated in the following: to develop options of forms of geokhod working body with geologic environment depending on parameters of generatrix of helicoid.

For development of the forms WB of the geokhod was accepted geometrical parameters of a helicoid into account the sizes of the created geokhod with roller working body for destruction of grounds of average hardness [13] $\mathrm{D}_{\mathrm{r}}=3,2 \mathrm{M}, \gamma=78^{0}, \mathrm{~h}_{\mathrm{B}}=0,8 \mathrm{~m}$ and width of generatrix is accepted equal $0,15 \mathrm{~m}$. Besides beams on which the directing WB settle down have been located at an some angle to pivot of the geokhod. The corner $y$ has been accepted: equal 90 angle degree $-\mathrm{a}$ direct form, $\mathrm{y}=78$ angle degree $-\mathrm{WB}$ takes a form of a right cone, $y=102$ angle degree - WB will be in the form of the back taper.

From the existing WB using for destruction of grounds with a hardness up to 1 on M.M. Protodyakonov's scale bar WB are the most suitable [12]. The received forms the helicoid surfaces of WB of the geokhod will be guides for the movement of cutter chain.

\section{Results and discussion}

Considering the requirements formulated in works $[7,10,13]$ and also conditions given above, we have received 21 forms of a guide of geokhod bar working body. Some options are presented in table 1 . The form of a guide of bar is allocated with the red line.

Table 1 - forms of a guide of geokhod bar working body 


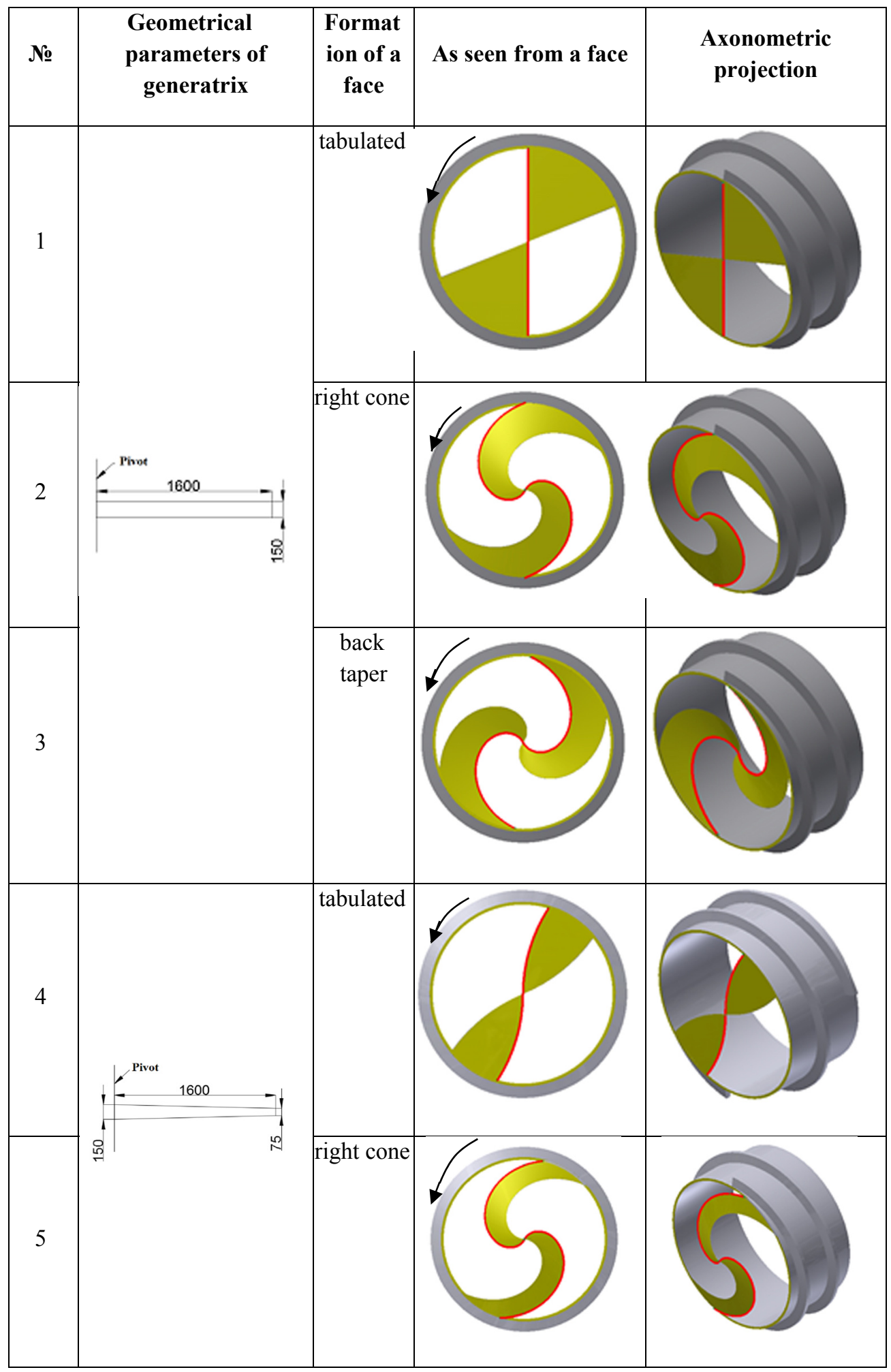




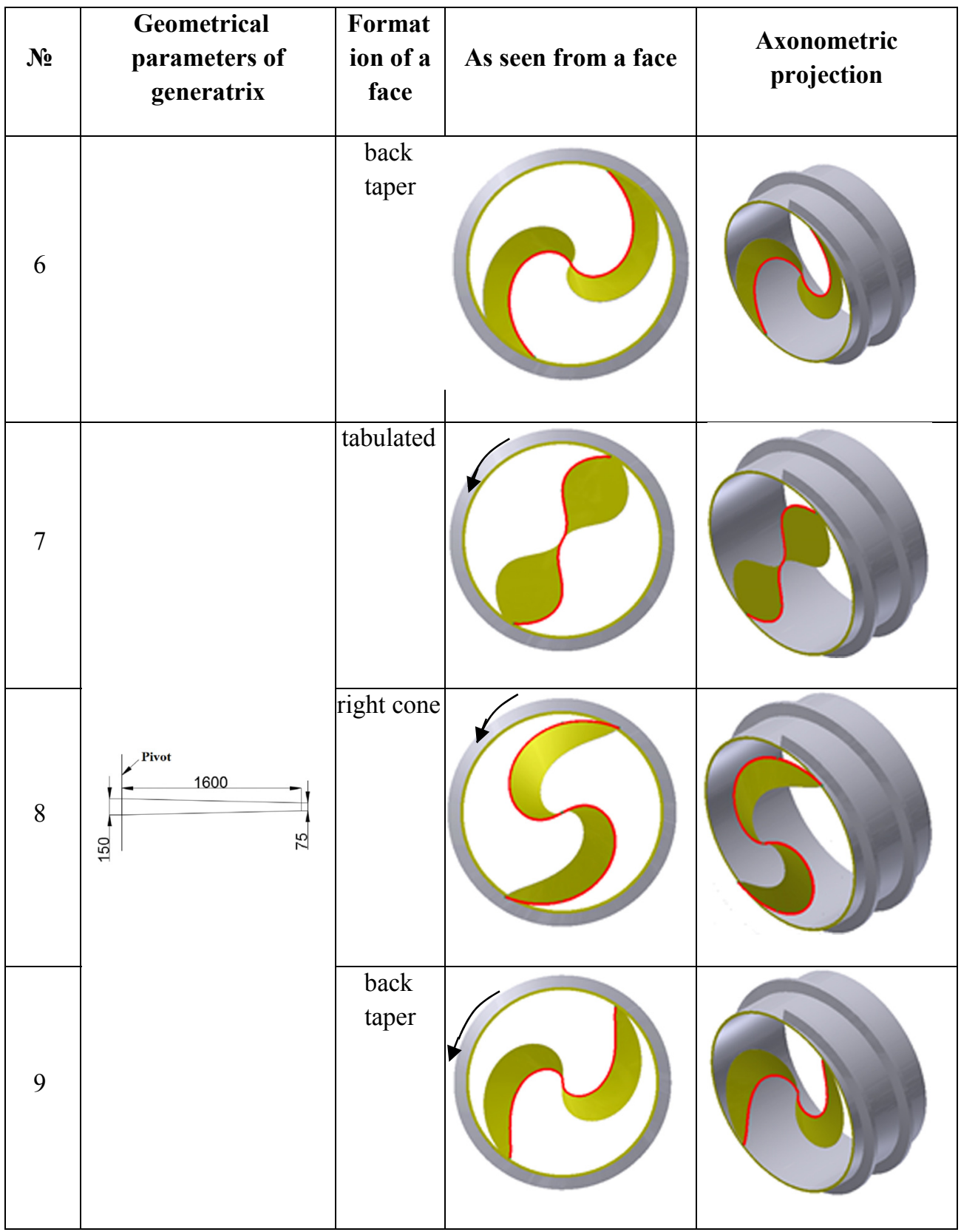

\section{Conclusions}

In table it is visible that on the form of a guide influences not only geometrical parameters generatrix of helicoid, but also angle of beam tilt WB to the frontal wall of a face (a face form). For reduction of forces of cutting and a possibility of self-tightening of the tunnelling equipment to the massif of grounds, a particular interest present forms of a guide of bar working body in the form of the back taper.

It is necessary for carrying out further researches: 
- to formulate requirements to geokhod bar working bodies for destruction of grounds with a hardness up to 1 on M.M. Protodyakonov's scale;

- to prove, using a finite element method, a rational form of a guide of bar working body.

- to receive dependences of a form of a guide of bar working body on diameter and a step of the screw blade of the geokhod.

\section{References}

1. G. Brierley, World Tunnel. And subsurface Excav, 9 (1998)

2. J. Carmody, R. Sterling. Underground space design (Reinhold, New York, 1993)

3. T. Wighman, ENR: News-Rec, 4, (1998)

4. S. Nishi, T. Seiki Mem. Sch. Eng. Nagoya Univ., 1 (1997)

5. R.V. Chernukhin, A.A. Dronov, M.Y. Blashchuk, IOP Conf. Ser.: Mater. Sci. Eng., 91, 012086 (2015)

6. A.B. Efremenkov, 6th International Forum on Strategic Technology (IFOST - 2011) 2:1, 348 (2011).

7. V.Y. Begljakov, V.Y. Timofeev, M.V. Dokhnenko, Appl. Mech. Mater. 682, 282 (2014).

8. M.Y. Blashchuk, A.A. Kazantsev, R.V. Chernukhin, Appl. Mech. Mater. 682, 418 (2014)

9. V. Aksenov, V. Sadovets, E. Rezanova, D. Pashkov, E3S Web Conf., 1503015 (2017)

10. V.V. Aksenov, A.B. Efremenkov, V.Y. Beglyakov, Appl. Mech. Mater. 379, 16 (2013).

11. M.Y. Blaschuk, A.A. Dronov, S.S. Ganovichev, IOP Conf. Ser. Mater. Sci. Eng., 127, 012033 (2016).

12. R.V. Chernukhin, M.Y. Blaschuk, V.Yu. Blumenstein, P.A. Chazov, IOP Conf. Ser. Mater. Sci. Eng., 142, 012003 (2016).

13. V.Y. Sadovets, V.Y. Beglyakov, A.B. Efremenkov, Appl. Mech. Mater. 770, 384 (2015). 